\title{
Sozialstaat in der Zerreißprobe - Perspektiven für die Frauen
}

Der Bruch der sozialliberalen Koalition, das Ende einer fast 13jährigen Epoche der bundesrepublikanischen Geschichte, kann nicht ohne Einfluß auf ein Editorial sein, das in die Problematik des Verhältnisses von kapitalistischer Akkumulation und Sozialstaat an Hand der folgenden Beiträge einführen soll. Diese Epoche war für die marxistische Linke nicht zuletzt auch eine Epoche der Diskussion um die Grenzen jener Reformpolitik, zu deren Verwirklichung die sozialliberale Koalition 1969 angetreten war. In deren Zentrum stand neben wirtschaftspolitischen Reformen, die aber bezeichnenderweise schon unter der Großen Koalition kodifiziert worden waren - eben jene Sozialpolitik, durch die nicht zuletzt auch Anteile am produzierten Neuwert massiv verschoben, aber auch die Mitbestimmungsmöglichkeiten der Betroffenen erweitert werden sollten. Das Ziel war (wenn auch durch die FDP-Beteiligung gedämpft) die Verwirklichung der »konkreten Utopie« des Godesberger Programms: die Schaffung einer menschlichen, solidarischen, von Chancengleich heit und Verteilungsgerechtigkeit geprägten Gesellschaft im Rabmen der freien Marktwirtschaft; einer Gesellschaft, die dann später u.a. von SPD-Wirtschaftsminister Schiller als "mündige Gesellschaft « charakterisiert wurde.

Eben diese mündige Gesellschaft muß sich heute von den 'Marktwirtschaftlern' sagen lassen, daß sie »über ihre Verhältnisse gelebt « habe; der »ausufernde Sozialstaat « habe ein »Anspruchsdenken « erzeugt, das dem "Leistungsgedanken « diametral entgegengesetzt sei; kurz: private Initiative und nicht zuletzt der Privatsektor überhaupt seien durch die Ausweitung des Staatssektors und die staatliche "Gängelung « nahe am Ersticken. Dagegen müsse jetzt so der rechte Wortführer dieser Politik, Graf Lambsdorff - »die Politik vor allem darauf ausgerichtet sein, dem Privatsektor in der Wirtschaft wieder mehr Handlungsspielraum und eine Zukunftsperspektive zu verschaffen; und innerhalb des Staatssektors muß sie die Gewichte von der konsumtiven in Richtung der investiven Verwendung verlagern. (FR v. 15.9.1982)

Schon in diesem kurzen Zitat wird deutlich, worum es jetzt den »Neokonservativen « in der Bundesrepublik geht: Um die Aufkündigung von in den Reformgesetzen seit 1969 festgeschriebenen Kompromißlinien, die nicht nur durch eine keynesianische Wachstumspolitik, sondern auch durch die weitere Integration und Institutionalisierung von sozialen Gruppen (insbesondere der Gewerkschaften) im politischen System abgesichert wurden. Die »neoliberale Konterrevolution «, deren politische und ideologische Grundlagen und deren (Miß-)Erfolge in verschiedenen Ausgaben der PROKLA bereits anhand von ausländischen Entwicklungen analysiert wurden, soll nun auch in der Bundesrepublik politisch als Allheilmittel gegen die ökonomischen und politischen Probleme im Verlauf der Weltwirtschaftskrise umgesetzt werden.

"Artisten in der Zirkuskuppel: ratlos - so könnte man in dieser Situation metaphorisch die Situation von Sozialisten und marxistischen Theoretikern (nicht nur) in der Bundesrepublik angesichts der neokonservativen und bürgerlichen Offensive seit Beginn der achtzi- 
ger Jahre umschreiben. Mit anhaltender Dauer und verschärfter Intensität der ökonomischen Entwicklungstendenzen ist unübersehbar geworden, daß es sich bei der mit der Weltwirtschaftskrise von 1974/75 eingeleiteten Entwicklungsphase nicht nur um einen vorübergehenden Abschwung, sondern um einen tiefgreifenden weltwirtschaftlichen Umstrukturierungsprozeß handelt, der weitreichende soziale und politische Dimzensionen aufweist. Mit der Verschlechterung der Verwertungsbedingungen von Kapital ist nicht nur der Akkumulationsprozess ins Stocken geraten; infrage gestellt ist ein gesellschaftliches Politikmodell, bekanntgeworden unter dem Namen »Wohlfahrtsstaat«, das den schon von Marx benannten Widerspruch von Kapitalismus und liberaler Demokratie scheinbar aufgehoben hatte. Der mit dem Namen Keynes verbundene gesellschaftliche Kompromiß zwischen den Klassen, wie er sich in nahezu allen entwickelten westeuropäischen Kapitalismen - wenn auch in unterschiedlicher Form - spätestens nach dem zweiten Weltkrieg herausbildete, konnte sich nahezu zwei Jahrzehnte auf einen hohen Beschäftigungsstand und stetig steigende Individial- und Soziallöhne stützen. Der fortschreitende Ausbau eines »Systems zur Reproduktion der Lohnarbeit«, von manchen als eine »de-commodification « der Ware Arbeitskraft interpretiert, machte vor allem die Sozialdemolsratie, als einem wichtigen Träger (wenn auch nicht "Erfinder «) dieses Gesellschaftsmodells vergessen, daß die Geschäftsgrundlage dieses Kompromisses in erster Linie ökonomisch (und das heißt immer noch: kapitalistisch) vermittelt war und ist. Stetige Akkumulation von Kapital und prosperierende Verwertungsbedingungen sind die Voraussetzungen dafür, daß der fiskalisch abhängige Steuer- und Sozialstaat Abschöpfungen vornehmen kann, um die wohlfahrtsstaatlichen Leistungen zu finanzieren. Mit der keynesianischen Wirtschaftspolitik schien dem Staat ein Instrumentarium der Intervention zugewachsen zu sein, die konjunkturellen Entwicklungsprozesse der Ökonomie mittels des »Mediums Geld« zu steuern und auf der Grundlage einer prinzipiellen Anerkennung des Privateigentums an Produktionsmitteln, also der DeThematisierung der Produktionssphäre, die Zirkulations- und Realisierungssphäre zu kontrollieren (vgl. dazu den folgenden Beitrag von J. Hoffmann).

Die mit diesen Entwicklungen einhergehende »Sozialstaatsillusion«(W. Müller/ Chr. Neusüß) wurde zwar schon frühzeitig von der marxistischen Linken zu analysieren versucht, doch mußten die dabei herausgearbeiteten »Grenzen des Sozialstaates" solange papierene Erkenntnisse bleiben, wie die prosperierende ökonomische Entwicklung und die Vollbeschäftigung der Arbeitskraft diese Grenzen kontinuierlich zu verschieben schienen. Als dann im Zuge der Weltwirtschaftskrise der siebziger und der anhaltenden Depression zu Beginn der achtziger Jahre immer deutlicher wird, daß die theoretisch bereits bestimmten Grenzen des Sozialstaates und der keynesianischen Wirtschaftspolitik konkrete Gestalt in Form hoher und dauerhafter Arbeitslosenzahlen und niedriger Wachstumsraten angenommen haben, wird allerdings auch deutlich, daß die veränderte ökonomische und politische Situation auch neue Fragen aufwirft, für die die »alten « Antworten nur beschränkte Orientierungshilfe geben können. Positive Strategieentwürfe der marxistischen Linken, wie die eingetretene Krise des Sozialstaates überwunden und der neoliberalen Marktoffensive erfolgreich entgegengetreten werden könnte, die in der heutigen politischen Situation unabdingbar sind, wenn die politische Offensive ergriffen werden soll, sind noch nicht entwickelt und liegen nicht vor. Die vor allem von den Gewerkschaften vertretene appellative Lösung, den Abbau des Sozialstaates zu verhindern und den Status quo zu sichern, ist eine genausowenig mobilisierende und perspektivische Parole wie die in der Linken vertretene Auffassung einer »Redimensionierung der abgeleiteten Bereiche« unter gewerkschaftlicher 
Kontrolle, um über eine Politik der Opfer entsprechende politische Gegenleistungen zur Verbesserung der Stärkeposition zu erringen. Beide Strategien, die sich zwar jeweils zu Recht gegen die wiedererstarkte Marktorthodoxie« der bürgerlichen Kräfte wenden, gehen an den Wurzeln der gegenwärtigen Misere vorbei.

Es ist zwar richtig, daran festzuhalten, daß das sich historisch entwickelte »System zur Reproduktion der Lohnarbeit «(Blanke) ein funktionales Strukturmerkmal hochentwickelter kapitalistischer Gesellschaften ist und deshalb auch im Zuge der neoliberalen Offensive, unterstellt man einmal »normale« parlamentarisch-demokratische Verhältnisse, nicht vollständig abgebaut werden wird. Allerdings darf diese Sichtweise nicht den Blick dafür verstellen, daß sich das gesamte gesellschaftliche Akkumulations»modell« der Nachkriegszeit in einem Umstrukturierungsprozess befindet, der noch nicht abgeschlossen ist. Die Anpassung der ökonomischen, politischen und sozialen Strukturen an die veränderten, weltmarktbestimmten Akkumulationsbedingungen von Kapital machen auch nicht vor funktionalen Strukturmerkmalen halt. Vielmehr ist (vgl. dazu den Beitrag E. Altvaters in diesem Heft) es gerade ein Merkmal solcher gesellschaftlicher Strukturbrüche, wie er mit der Krise von 1974/75 eingeleitet wurde, funktionale Bestandteile des überkommenen Akkumulationsmodells umzumodellieren und erneut verwertungskompatibel zu machen. Wird diesen bestandsnotwendigen Entwicklungsprozessen keine politische Alternative entgegengesetzt, sondern werden diese Prozesse nur zu effektivieren versucht (wie dies die Sozialdemokratie in der Bundesrepublik im Zuge ihrer korporativen Modernisierungsstrategie im Bündnis mit weltmarktorientierten Kapitalen und den Gewerkschaften zu praktizieren versucht hat), dann geht damit auch die Gefahr einher, selbstt zum »Opfer dieser Restrukturierungen « $z u$ werden. Das Ende der sozialliberalen Koalition ist ein Musterbeispiel für eine solche Entwicklung: Als »Modell Deutschland« auch international bekannt, berühmt und manchmal berüchtigt geworden, hat die Sozialdemokratie versucht, durch Förderung der technologischen Entwicklung und der Rationalisierung der Produktionsprozesse und -strukturen die Entwertungsprozesse zu forcieren, um einen sozialpolitisch und konsensual abgesicherten gesellschaftlichen Restrulsturierungsprozess in Gang zu setzen. Mit anhaltender Dauer der wirtschaftlichen Depression, dauerhaft hohen und von Krise zu Krise steigenden Arbeitslosenzahlen sowie schnell anwachsenden staatlichen Defiziten ist es mit dieser Strategie aber immer weniger gelungen, die »sozialen Umstellungskosten « zu monetarisieren. Die scheinbaren Grenzen der staatlichen Verschuldung mussten als Begründung herhalten, warum die Opfer der Restrukturierung auf eine immer geringere staatliche Unterstützung vertrauen konnten. Die mit dem Keynesianismus verknüpfte politische Filterung der ökonomischen Entwicklungsprozesse wurde immer mehr zugunsten einer Ökonomisierung politischer Entscheidungen verdrängt: Der Marlst verdrängte das politische Prinzip der Intervention. Spätestens mit der Operation ' 82 wurde deutlich, daß der hinter der sozialliberalen Koalition existierende gesellschaftliche Konsens weitere Einschnitte in das soziale Netz nur schwer würde verkraften können. Eingezwängt in die »ökonomischen Sachzwänge« und ohne eigenständige politische Perspektiven, sondern lediglich dem alten Politikprojekt der Vor-Krisenphase verhaftet, kam die SPD über die Forderung nach einer sozial ausgewogenen Komponente im Rahmen des Austerity-Programms i.S. der Forderung nach einer (zeitlich begrenzten) Arbeitsmarktabgabe für "Besserverdienende« nicht hinaus. Die ablehnende Haltung der Freien Demokraten und das nachgeschobene "Lambsdorff «-Papier, das von der Sozialdemokratie als Provokation empfunden werden musste, waren deshalb lediglich Anlaß, nicht Ursache des Bruchs der Koalition. 
Diese Ursachen werden in dem einleitenden Beitrag von Jürgen Hoffmann auf dem Hintergrund der historischen Entwicklung seit 1969 untersucht: Im Zentrum steht dabei die (schon im "Sozialstaatsillusions«-Aufsatz von Wofgang Müller und Christel Neusüß 1970 aufgeworfene) Frage, inwiefern eine Reformstrategie, die über wirtschaftliches Wachstum und dessen staatliche Regulierung einen gesellschaftlichen Konsens produzieren bzw. stabilisieren will, unter Bedingungen privatkapitalistischer Eigentumsverhältnisse (der "Autonomie der Unternehmen «) Erfolg haben kann. Der Autor zeigt in seiner Untersuchung (die besonders im ersten T'eil die Ergebnisse der Diskussion um die Akkumulations- und Staatstheorie in der PROKLA zusammenfaßt), daß aufgrund des Primats wirtschaftlichen Wachstums als Voraussetzung der sozialdemokratischen Politik diese mit den Veränderungen der ökonomischen und gesellschaftlichen Strukturen (das o.a. veränderte Akkumulations» modell«) sich in einer Weise mit verändern muß, daß die ursprünglichen Reformziele z.T. geradewegs in ihr Gegenteil verkehrt werden. Der Artikel endet mit perspektivischen Aussagen zu Möglichkeiten der Entwicklung eines neuen, an der Reproduktion der natürlichen und menschlichen Ressourcen der Gesellschaft orientierten Konsenses, die gerade nach dem Bruch der sozialliberalen Koalition und angesichts der letzten Wahlergebnisse in Hamburg und Hessen an Bedeutung gewinnen könnten.

Die soziale Sicherung wird in dem Artikel von J. Hoffmann als ein Eckstein neben anderen analysiert, die mit den veränderten ökonomischen Strukturen und den daraus sich scheinbar »sachnotwendig" ergebenden Eingriffen in die Staatsausgaben geschliffen werden. In den weiteren Beiträgen stehen die soziale Sicherung und deren Voraussetzungen im Zentrum der Analysen, hier wiederum zugespitzt auf die Perspektiven für die Frauen. Bereits in den vergangenen Jahren seit 1974/75 wurden im Zuge der "Konsolidierungsmaßnahmen« die Reformprogramme im Bereich der Arbeitnehmerschutz-, der Sozialversicherungs- und der Wohnungspolitik, der Arbeitsmarktpolitik, dem Kündigungsschutz, der Betriebsverfassungspolitik, der Familienpolitik und der Bildungspolitik demontiert. Ging es in den bisherigen politischen und sozialen Kämpfen noch darum, Reformprinzipien des sozialdemokratischen Wohlfahrtsstaats mit seinen Leitbildern, 'Chancengleichheit' und 'soziale Gerechtigkeit' auch zum Teil gegen die Sozialdemokratie aufrechtzuerhalten, dürfte in den kommenden Jahren die Kernstruktur des Systems sozialer Sicherung, das Versicherungssystem verstärkt in die Diskussion geraten. Schon heute ist es so, daß das in den 50er Jahren - entsprechend dem liberalistischen Sozialpolitikverständnis - aufgebaute System sozialer Sicherung, mit dem Prinzip der Stärkung der »Freiheit« und »Verantwortung" des Einzelnen, eine gänzlich andere Funktion erhalten hat. Beispielsweise muß bereits jetzt die Sozialhilfe für eine wachsende Zahl von Personen bzw. Familien die materielle Grundsicherung, das 'historisch-moralische Existenzminimum' erbringen. Damit hat sie faktisch primär die Sicherung eines minimalen Einkommens für Personengruppen übernommen, die durch das angeblich engmaschige System sozialer Sicherung sonst nicht abgesichert sind. Die Lücken im System sozialer Sicherung, die der Sozialhilfe im vergangenen Jahrzehnt bereits ein völlig anderes Aufgabengebiet zugewiesen haben, verweisen darauf, daß es sich nicht um bloße Übergangsprobleme handelt, sondern Ausdruck der tiefgreifenden ökonomischen Krise sind. - Insbesondere bei jüngeren Hilfeempfängern sind überproportionale Steigerungsraten zu konstatieren; die größten Zuwachsraten bei den Empfängern von Hilfe zum Lebensunterhalt sind seit 1974 festzustellen; die Quote der Haushalte mit Bezug von Hilfe zum Lebensunterhalt, die gleichzeitig Arbeitslosengeld bzw. -hilfe bezogen, stieg seit 1972 beständig an. Und hier dürften die offiziellen Zahlen der Arbeitslo- 
senstatistik noch erheblich täuschen: Der Anteil der Arbeitslosen unter den Sozialhilfeempfängern ist mit Sicherheit erheblich höher als ihn die Statistik ausweist, da ein großer Teil der Sozialhilfeempfänger Frauen sind, die keine Unterstützung vom Arbeitsamt beziehen bzw. nicht arbeitlos gemeldet sind, obwohl sie arbeitslos sind. Diese brüchige Erwerbsorientierung, trotz steigenden Anteils erwerbstätiger Frauen an der Erwerbsbevölkerung, die sich im Falle von Arbeitslosigkeit immer wieder manifestiert, unterstreicht die Sonderrolle, die Frauen in der Gesellschaft immer noch spielen. Sie ist bestimmt durch eine vergleichsweise schlechtere Ausbildung, durch ein verengtes Spektrum beruflicher Möglichkeiten und durch eine geschlechtsspezifische Ausgangssituation auf dem Arbeitsmarkt. Gleichzeitig ist sie gekennzeichnet durch widersprüchliche Lebensperspektiven und durch eine zum Teil starke materielle Abhängigkeit von familienabhängigen Versorgungsleistungen, die, als Hinterbliebenenrente oder als eigenständig erworbener Rentenanspruch aufgrund der Lohndiskriminierung von Frauen eine Unterversorgung bis ins hohe Alter mit sich bringen. Diese durch Arbeitssituation, Lohndiskriminierung, Sozial- und Rentenversicherungsrecht beständig reproduzierten schlechten Lebensverhältnisse eines Teils der Frauen nehmen aufgrund der steigenden Arbeitslosigkeit, die Frauen überproportional betrifft, noch zu.

Die anhaltende Wirtschaftskrise hat bereits in den 70er Jahren dazu geführt, daß die Bevölkerung in verschiedene gesellschaftliche Bereiche gespalten wurde - jedoch hauptsächlich unterschieden durch Erwerbstätigkeit und Arbeitslosigkeit. Insbesondere Frauen und Jugendliche sind im vergangenen Jahrzehnt von den Folgen der ökonomischen Krise und der Demontage der sozialliberalen Reformvorhaben v.a. in den Bereichen der Berufsbildungs-, Arbeitsmarkt-, der Arbeits- und Sozialpolitik betroffen worden. Im Gegensatz zur dramatisch sich zuspitzenden Jugendarbeitslosigkeit wird der sich vergleichsweise zuspitzenden Frauenarbeitslosigkeit kaum verstärkte Aufmerksamkeit geschenkt. Im Gegenteil! Die direkte und indirekte Ableitung der weiblichen Erwerbsquote - d.h. die Bestimmung ihres jeweiligen relativen Anteils - aus den jeweiligen politisch-ökonomischen Rahmenbedingungen und einer sie flankierenden Gesetzgebung, scheint sich im historischen Verlauf immer wieder als »Normalisierungsbewegung « durchzusetzen (vgl. I. Wegehaupt-Schneider in diesem Heft). Und zwar ungeachtet des Widerstands, den Frauen ihrer sozialen und politischen Diskriminierung, vor allem auch in den vergangenen dreizehn Jahren, verstärkt entgegensetzten.

Wir haben deshalb in diesem Heft versucht, auf dem Hintergrund einer Bestandsaufnahme der Situation der erwerbstätigen Frau, die Lebensperspektiven dieser von der sozialen Demontage am stärksten betroffenen Gruppe zur Diskussion zu stellen:

Ursula Westphal-Georgi zeigt in ihrem Artikel die Gefahr auf, die sich mit dem Abtreten des Wohlfahrtsstaates sozialdemokratischer Prägung speziell für Frauen ergibt: Ohne kompensatorische sozialstaatliche Maßnahmen dürfte sich die geschlechtsspezifische Arbeitsteilung rigide verschärfen und die Lebensperspektive junger Frauen wieder auf die Tätigkeit als Hausfrau und Mutter verengen. Konzepte, die die Lücken sozialstaatlicher Maßnahmen schließen sollen, stünden mit den bewährten familienpolitischen Maßnahmen aber auch mit den Überlegungen zur 'Dualwirtschaft' schon bereit. Entgegen diesen Konzepten und entgegen der frauenspezifischen Autonomisierungsstrategie Claudia von Werlhofs' werden frauenpolitische Strategien favorisiert, die auf eine offensive Weiterentwicklung berufs-, arbeitszeitpolitischer- und sozialpolitischer Konzepte setzen, mit dem Ziel, familienunabhängige Orientierungen zu stabilisieren. Ingeborg Wegehaupt-Scbneider kommt in ihrer histo- 
risch angelegten Untersuchung zu dem Ergebnis, daß Frauenerwerbsarbeit in der Vergangenheit schon immer als Zuverdienertätigkeit begriffen wurde, die die Entwicklung einer familienunabhängigen Lebensperspektive behinderte. Sie weist im einzelnen nach, daß beide - Sozialpolitik und familienpolitische Maßnahmen - von der Doppelfunktion der Frau in Familie und Erwerbstätigkeit ausgehen und sich in ihrer jeweiligen Durchsetzbarkeit den Gegebenheiten des Arbeitsmarktes anpassen. Diese politischen Anpassungsstrategien führen im historischen Verlauf dazu, daß es immer wieder zu einer »Normalisierung« des relativen Anteils erwerbstätiger Frauen kommt sowie zu einer verfestigten Abgrenzung von männlichen und weiblichen Tätigkeiten, die einen Verdrängungswettbewerb zuungunsten männlicher Arbeitskräfte gar nicht erst entstehen läßst. Lothar Lappe beschreibt in der anschließenden Untersuchung die strukturellen Barrieren, die eine Konkurrenz zwischen Männern und Frauen auf dem Arbeitsmarkt verhindern. Er kommt zu dem Ergebnis, daß spezifische, institutionelle Regelungen der Qualifikation, Belastung und Gratifikation eine verschärfte Arbeitsplatz- und Lohndiskriminierung der arbeitenden Frauen bewirken. Indem die weiblichen Erwerbstätigen auf wenige unattraktive Arbeitsbereiche abgedrängt werden, indem sie bei unterdurchschnittlicher Bezahlung starke Qualifikationsreduzierungen erfahren und mit erheblichen Belastungen konfrontiert werden, zeigt sich die für den geschlechtsspezifischen Teilarbeitsmarkt typische wechselseitige Verstärkung negativer Segmentationsfolgen, die die Arbeitsmarktchancen erwerbstätiger Frauen nachhaltig verschlechtern. Die st aatliche Arbeitsmarktpolitik sowie die betriebs- und tarifpolitischen Aktivitäten der Gewerkschaften haben bisher diese Arbeitsmarktsegmentation in männliche und weibliche Bereiche durch ihre einseitige Orientierung auf den fachspezifischen (männlichen) Arbeitsmarkt zusätzlich verstärkt. Andrea Ruby, Brigitte Göttgens, Sigrid Koeppinghoff nehmen in ihrem Aufsatz das Zusammentreffen der Benachteiligung der Frau im Erwerbsleben mit der der Familienfixierung geschuldeten unstetigen Erwerbs- und Versicherungsbiographie zum Ausgang ihrer Analyse der Rentenreform '84. Sie beschreiben zum einen die Leistungsfähigkeit und die Defizite der gegenwärtigen Altersicherung der Frau; zum anderen messen sie die Rentenreform an der gèsellschaftlich definierten Rolle der Frau (auf dem Arbeitsmarkt), um Tragweite und Implikation der rentenrechtlichen Bestimmungen adäquat einschätzen zu können. Sie kommen zu dem Ergebnis, daß es sich bei der Rentenreform ' 84 um eine versicherungsimmanente Förderung einer familienbezogenen Frauenrolle handelt (Subventionierung der Hausfrauenehe), da sie gerade diejenigen Verhältnisse festschreibt, die die unzureichende Alterssicherung der Frau begründen: unterschiedliche Ansprüche von Männern und Frauen im Hinterbliebenenfall und keine nachträgliche Korrektur der Lohndiskriminierung in Frauenlohngruppen. Hedwig Rudolph diskutiert das Ineinandergreifen familien- und sozialpolitischer Diskriminierung als "patriarchale Strukturen des Sozialstaates«, die immer wieder eine Sonderbehandlung der Frau hervorbringen, die das "Arbeitnehmerpatriarchat « in den Gewerkschaften mit seiner Tabuierung des Normalarbeitstags noch verstärkt: Da die praktizierte Neuverteilung der Arbeitszeit als Teilzeitarbeit und Job-Sharing von den Gewerkschaften bisher nicht verhandelt wurde, setze sich diese Arbeitszeitverkürzung naturwüchsig durch und verschlechtere damit unkontrolliert Arbeitssituation und Arbeitsmarktlage der Frauen. Die zusätzliche Konfrontation der Frauen mit sich verengenden Planungshorizonten treibt sie innerhalb ihres Rahmens von betrieblichen und außerbetrieblichen Lebensbedingungen zu ganz spezifischen privaten Widerstandsformen. Dies allerdings verweist auf die Notwendigkeit, so schließt der Artikel, einer strukturellen Neuverteilung von Lohnarbeit und Hausarbeit. 
Mit den Artikeln von Elmar Altvater und Michael Krätke wird der Kreis zum Bereich Sozialpolitik geschlossen. Elmar Altvater nimmt die eingangs zitierten Angriffe gegen die "Auswüchse« des Sozialstaats und die dagegen propagierten neo-konservativen Allheilmittel zum Anlaß, die Abhängigkeit der sozialstaatlichen Entwicklung von der Akkumulation des Kapitals zu diskutieren. In diesem Zusammenhang werden von ihm die sozialen Funktionen ( $=$ Stabilitätsleistungen) der sozialen Sicherung herausgearbeitet und es wird in einer historischen Betrachtung der gegenwärtigen Krise die von uns oben schon angeschnittene Frage aufgeworfen, inwieweit wir es heute nicht mit einem krisenhaften Strukturbruch der Formen der gesellschaftlichen Reproduktion, mithin auch der Form des Sozialstaats, zu tun haben. Michael Krätke diskutiert anhand neuerer - insbesondere angelsächsicher - Literatur den Begriff des Sozial- bzw. Wohlfahrtsstaats und versucht, ausgehend von der Marxschen Kritik der politischen Ökonomie und darin enthaltener Leerstellen in bezug auf eine nähere Bestimmung des »Werts der Ware Arbeitskraft«, Elemente einer Theorie des Sozialstaats. $\mathrm{zu}$ entwickeln.

Gerade das Ende der sozialliberalen Koalition wirft jedoch noch einmal die Frage auf, ob der zu erwartende Umstrukturierungsversuch der Politik der sozialen Sicherung auch das Ende offensiver gesellschaftlicher Reformvorstellungen bedeuten muß. In bezug auf die gesellschaftliche Stellung der Frau ist das 'Rad der Geschichte' sicherlich nicht mehr so ohne weiteres zurückzudrehen. Obwohl die Frauen bisher nahezu ausschließlich verwiesen sind auf schlechte, unsichere Arbeitsplätze, mit geringer Entlohnung, beständiger Bedrohung durch Rationalisierung und rentenmäßiger Unterversorgung im Alter, hat die weibliche Erwerbsquote seit den 60er Jahren beständig zugenommen. Dies ist gleichzeitig verbunden mit einer Zunahme erwerbstätiger Ehefrauen von sowohl Arbeitern und Angestellten. Das heißt, trotz abnehmender Zahl von Arbeitsplätzen (und trotz beklagenswert schlechter Arbeitsbedingungen) steigt die Frauenbeschäftigung absolut an. Gleichzeitig nimmt die Umschichtung von den selbstständigen und mithelfenden Familienangehörigen zu den abhängig Beschäftigten zu. Diese steigende Arbeitsplatznachfrage trifft zusammen mit besonders starken Geburtenjahrgängen in den 80er Jahren, die von dem Ausbildungsbereich in den Beschäftigungsbereich übergehen und der Tatsache, daß Frauen eine zunehmende Orientierung auf berufliche Tätigkeit aufweisen, die weit über das Interesse an "bloßem Geldverdienen' hinausgeht. Daß soziale Identität untrennbar verknüpft ist mit der gesellschaftlichen Verausgabung von Arbeit, ist vor allem für Mädchen und junge Frauen zur Selbstverständlichkeit geworden. Dies zeigt sich zum einen darin, daß seit Anfang der 70er Jahre unter den jungen Frauen deutlich die Gruppe der Ungelernten abgenommen hat bei gleichzeitigem Anstieg der Anteile junger Frauen bei mittleren Bildungsabschlüssen. In bezug auf Mädchen und junge Frauen kann man durchaus von einer »Bildungsexplosion« sprechen, auch wenn die Aufbruchsforderung der 60er Jahre »Chancengleichheit durch Bildung « für Frauen am wenigsten umgesetzt wurde, wie die Existenz des abgeschotteten weiblichen Areitsmarkts beweist. Zum anderen ging mit dem Anstieg des Bildungs- und Ausbildungsniveaus der Frauen in den vergangenen 20 Jahren und mit der Zunahme der weiblichen Erwerbstätigkeit auch ein beständiges Anwachsen des sozialen und politischen Engagements einher - erinnert sei hier nur an den Kampf um die Reform des $\$ 218$. Obwohl dieser Aufbruch von Frauen von beständigen Rückschlägen und Desillusionierungen begleitet ist, wie es ihre Situation auf dem Arbeitsmarkt, ihre Doppelbelastung durch Familie und Beruf und die die gesellschaftlichen Diskriminierungen wieder verstärkenden Entwicklungen im Sozial- und Rentenversicherungsrecht belegen, sind sie doch besser auf 
ihre Zukunft vorbereitet als frühere Generationen. Ihre leitende Orientierungsgröße ist nicht mehr ausschließlich die Familie, sondern die Vereinbarkeit von Beruf und Familie. Damit ist jedoch die 'Zukunft der Frauenarbeit' nicht mehr in der privaten Idylle befangen. Der Aufbruch der Frauen, der sich seit den 60er Jahren langsam und kontinuierlich vollzog, wird deshalb auch - trotz anhaltend schlechter Arbeitsmarktlage und manipulativ angewandter Familien- und Sozialpolitik - nicht mehr rückgängig zu machen sein. Zwar belegen die aktuellen Versuche der »Féinsteuerung« des Arbeitsmarktes die erneut eingeleitete "Normalisierung« des relativen Anteils der arbeitenden Frauen: durch die

- Erhöhung der Kindergartentagessätze,

- Senkung der Unterhaltssätze bei Fortbildung und Umschulung,

- administrative und finanzielle Behinderung von Modellversuchen zur Nachqualifizierung arbeitsloser Frauen,

- frauenspezifische Definition der »Verfügbarkeit « für die Arbeitsvermittlung als Voraus" setzung für die Inanspruchnahme von Leistungen.

Ein völliges Herausfallen der Frauen aus dem Arbeitsmarkt dürfte jedoch schon aufgrund der relativen Eigenständigkeit ihres Arbeitsmarktes nicht möglich sein. Dieser Umstand, sowie der sich verstärkende ökonomische Zwang zur Lohnarbeit, als zwingende materielle Grundsicherung der Familie, und die Zunahme eigenständiger Lebensperspektiven der Frauen werden dazu führen, daß die Zukunft der Frauenarbeit nicht einfach zur abgeleiteten Größe der wirtschaftlichen Entwicklung und der 'Finanzkrise des Staates' wird. Diese Zukunft wird aufgrund dieser Bedingungen in der widersprüchlichen Veränderung beruflich strukturierter Arbeit liegen, als Veränderung der alltäglichen privaten Verhältnisse und als Weiterentwicklung arbeits-, arbeitszeitpolitischer sowie sozialer Verhältnisse, die sowohl die Frauen als auch die 'Berufe' verändern werden.

Bisher ist die Forderung nach Aufhebung der Trennung von Kopf- und Handarbeit nur allgemein erhoben worden; für Frauenarbeit und Frauenerwerbsarbeit muß sie überhaupt erst gestellt werden. Um hierzu einen Beitrag zu leisten, haben wir vor, künftig Probleme der Frauenarbeit und Frauenerwerbsarbeit kontinuierlich aufzunehmen, um die Möglichkeit zu schaffen, die gesellschaftlichen Verhältnisse, in denen Frauen leben und arbeiten, zur Diskussion zu stellen.

Die Diskussion, die Peter Brandt und Günter Minnerup mit ihrem Beitrag in Heft 47 der PROKLA zur "nationalen Frage eröffnet haben, wird in diesem Heft fortgesetzt. Die dort von den beiden Autoren angesprochenen Fragen werden in der linken Öffentlichkeit sehr kontrovers diskutiert. Frank Dingel fühlte sich durch die von Brandt/Minnerup vertretene Position zu einer scharfen Polemik herausgefordert, auf die die beiden angegriffenen Autoren geantwortet haben. Wir veröffentlichen diese Kontroverse, weil wir meinen, daß die Diskussion über die Bedeutung der "Nation« und der »nationalen Frage« gerade erst in der Linken begonnen hat und deshalb auch noch keine »abgeklärten« Resumées dazu zu erwarten sind. Die beiden hier vertretenen Positionen scheinen unvereinbar, kennzeichnen aber auch die Bandbreite innerhalb der Diskussion unter Linken. Wir hoffen, daß durch die Schärfe der hier abgedruckten Kontroverse die Konturen dieses Gegensatzes deutlich hervortreten und die Fortführung der Diskussion erleichtert - und nicht verschüttet - wird! 